\title{
Role of Crack-Tip Residual Stresses in Stress Corrosion Behavior of Pre-stressing AA7020
}

\author{
Ashish Thakur* \\ Department of Mechanical Engineering, Solid Mechanics and Design Chair, Ethiopian Institute of Technology, School of Mechanical and \\ Industrial Engineering, Mekelle University, Mekelle, Ethopia
}

Received 08 March 2019, Accepted 11 May 2019, Available online 13 May2019, Vol.9, No.3 (May/June 2019)

\begin{abstract}
This paper analyzes stress corrosion cracking (SCC) of pre-cracked samples in the presence of compressive residual stresses generated in the vicinity of the crack tip during fatigue pre-cracking. Research focuses on the role of cracktip residual stresses of compressive nature, generated by fatigue loading, in stress corrosion cracking of pre-cracked samples of medium high strength aluminum alloy 7020 subjected to localized anodic dissolution and hydrogen assisted cracking. Fatigue pre-cracking load on the samples generates compressive residual stresses in the vicinity of the crack tip which improve the stress corrosion behavior of the aluminum alloy by delaying either the metal dissolution or the hydrogen entry, thus increasing the fracture load in an aggressive environment. The rice model of the residual stress distribution in the vicinity of a crack tip may be usedto explain these retardation effects by estimating the stress level and plastic zone size. Microscopically, compressive residual stress produce a transition topography between the fatigue pre-crack and the cleavage-like (unstable) fracture mode.
\end{abstract}

Keywords: Stress corrosion cracking; crack-tip residual stresses; pre-stressing aluminum alloy, microscopical, fracture

\section{Introduction}

It is well known in engineering fracture mechanics that overloads produce a retardation effect on fatigue crack propagation (Broek D. et al, 1982; Barsom J.M. et al, 1987; Suresh S. et al, 1991). The effects of overloads on environmentally assisted cracking or, in traditional form, stress corrosion cracking have been studied in previous works (Hanisch A.H. et al, 1982) dealing with the initiation of a stress corrosion crack, the main conclusions being that overloads increase the threshold level or delay the time for initiation of see cracks (increasing the incubation period).

During last years the use of pre-cracked samples has become widespread in stress corrosion testing (Toribio J. et al, 1993). These specimens are quite realistic and favor the localization of the environmental attack just at the crack tip. In addition, they can be easily prepared by fatigue, and therefore machining is not required.

There is, however, an important effect of fatigue pre-cracking of the samples: the generation of compressive residual stresses in the vicinity of the crack tip, produced by strain compatibility in the subsequent plastic zone. These stresses may play an

*Corresponding author's ORCID ID:0000-0001-9287-700X DOI: https://doi.org/10.14741/ijcet/v.9.3.7 important if not decisive role in the overall SCC process.

The fracture mechanics approach to the stress corrosion cracking (SCC) phenomenon in metals and alloys requires the performance of stress corrosion tests on pre-cracked specimens (ISO 7539-6 et al, 1989; BranchoC.M. et al, 1977) in which a pre-crack must be produced by fatigue, creating compressive residual stresses in the vicinity of the crack tip. These stresses are generated by strain compatibility in the subsequent plastic zone and may play a relevant role in the overall SCC process. From the microscopical point of view, previous research showed that crack-tip residual stresses produce in the middle high strength aluminum alloy an environmentally assisted transition topography between the fatigue pre-crack and the cleavage-like (unstable) fracture mode. Such a transition zone has a specific microscopic appearance depending on the environmental mechanism under consideration.

This work analyzes the mechanical aspects of the phenomenon, so the stress field produced by the cyclic loading is modelled, and the extension of the plastic zone compared with the sizeof the environmentally assisted region. Thus the present work offers the combined microandmacro-approach to the phenomenon which is original and innovative from the 
material science point of view, since microscopic modes of fracture associated with stress corrosion cracking(micro-approach) are related to stress state and plastic zone evolution (macro-approach in the continuum mechanics sense) calculated by means of a simple model, which allows an easy estimation of values directly applicable to a wide range of stress corrosion processes in which a more accurate computation of stress-strain evolutions in the vicinity of a crack tip often requires very detailed finite element analyzes for different specimen geometries and crack configurations and these procedures are rather costly and time consuming.

\section{Experimental procedure}

The experiments were performed on a hot rolled medium high strength aluminum alloy, supplied in plate form of $5 \mathrm{~mm}$ thickness, whose chemical composition and mechanical properties appear respectively in the Table 1 and Table 2(Thakur A. et al, 2007). The specimen was prepared according to ASTM E-399 standard. In this experiment, a cantilever beam specimens was used, the dimensions of which is shown in Figure 1. All tests were conducted at Metallurgical and Mechanical Engineering Department, IIT Bombay. The specimen contained a sharp $\mathrm{V}$ notch and was precracked by fatigue. Pre-cracking of the samples was carried out in an air environment. Various series of samples were prepared using different maximum values of the fatigue pre-cracking load during the last step (just previous to the fracture test), so as to analyse the effect of the maximum load on the ulterior stress corrosion cracking behaviour of the aluminum alloy.

Table 1: Chemical composition of the aluminum alloy (masses \%)

\begin{tabular}{|c|c|c|c|c|c|c|c|c|}
\hline $\mathrm{Zn}$ & $\mathrm{Mg}$ & $\mathrm{Si}$ & $\mathrm{Mn}$ & $\mathrm{Zr}$ & $\mathrm{Ti}$ & $\mathrm{Cr}$ & $\mathrm{Cu}$ & Balance \\
\hline 4.35 & 1.4 & 0.012 & 0.22 & 0.059 & 0.012 & 0.032 & 0.06 & 93.885 \\
\hline
\end{tabular}

Table 2: Mechanical properties of the aluminum alloy 7020

\begin{tabular}{|c|c|c|c|c|c|}
\hline $\mathrm{E}(\mathrm{GPa})$ & $\sigma_{\mathrm{y}}(\mathrm{MPa})$ & UTS(MPa) & $\begin{array}{c}\text { Elongation } \\
(\%)\end{array}$ & $\begin{array}{c}\text { Reduction } \\
\text { in area } \\
(\%)\end{array}$ & $\mathrm{K}_{\mathrm{IC}}\left(\mathrm{MPa}^{\mathrm{N}}{ }^{1 / 2}\right)$ \\
\hline 69 & 280 & 383.8 & 12 & 21 & 26.91 \\
\hline
\end{tabular}

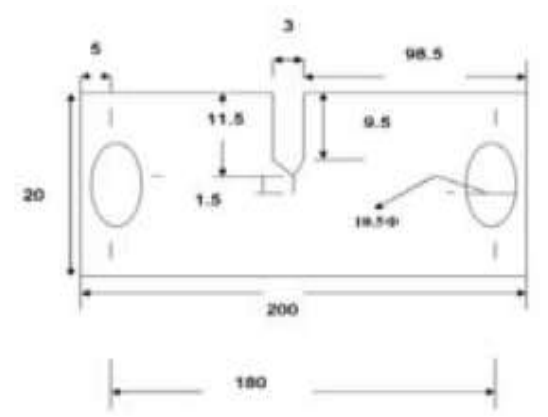

Fig. 1.Cantilever Beam specimen dimensions. (All dimensions are in $\mathrm{mm}$ )
This section analyzes the consequences of cyclic residual stresses in the development of SCC. Emphasis is placed on the effect of the maximum stress intensity factor during the last stage of the fatigue pre-cracking $\left(K_{\max }\right)$ on the posterior stress corrosion behavior of the material. To this end, four different pre-cracking programs were designed with $K_{\max } / K_{\mathrm{IC}}=0.58,0.69,0.71,0.76$ and 0.94 , where $K_{\mathrm{IC}}$ is the fracture toughness, so the Pre-damages in the samples reaches several relations to the critical value for fracture in air. Samples were subjected to slow strain rate testing (ParkinsR.N. et al, 1993). The crosshead speed was $8.0 \times 10^{-8} \mathrm{~ms}^{-1}$, followed by previous experience (ISO 7539-7 et al, 1989). The aggressive environment was an aqueous solution of $1 \mathrm{~g} / 1 \mathrm{Ca}(\mathrm{OH})_{2}$ $+0.1 \mathrm{~g} / 1 \mathrm{NaCl}+\mathrm{H} \mathrm{Cl}$. All tests were carried out under potentiostatic control, covering a broad range from $\mathrm{E}=$ $-1000 \mathrm{mV}$ SCE to $\mathrm{E}=-100 \mathrm{mV}$ SCE. The SCC tests were done at rising load. The SCC experiments were cantilever beam tests (Bayoumim.R. et al, 1996; Mulherinj.H. et al, 1967) in aqueous solution with samples of hot-rolled aluminum alloy 7020 previously pre-cracked. Since a certain period is required for a crack to initiate, pre-cracked specimens are used in $\mathrm{K}_{1 \mathrm{SCC}}$ determination. Maximum time was kept to analyze the SCC growth and threshold stress intensity was 388 hour's. Table 1 and Table 2offer respectively the chemical composition and mechanical properties of the aluminum alloy 7020. The experimental details are given in reference (Bayoumim.R. et al, 1996).

Susceptibility to the SCC was measured through the ratio of the failure load in an aggressive environment to that in the air, as recommended by the ISO Standard. An explained in the detail in Ref. (Toribio J. et al, 1996), there are two regions of the potential in which the effect of the environment reduces the fracture load: the highest potential correspond to the anodic regime, and the mechanism is the localized anodic dissolution (LAD), whereas the lowest potentials are related to the cathodic regime, and the environmental mechanism isthe hydrogen assisted cracking (HAC). Figure 2 and Figure 3 show the experimental results in a plot representing the failure load in solution Fc (divided by the reference value in air Fo)as a function of the maximum stress intensity factor during the last stage of the fatigue pre-cracking $K_{\max }$ (divided by the fracture toughness $K_{\text {IC }}$ ). For both LAD and HAC, the susceptibility to the environment decreases (i.e. the fracture load increases) as the maximum fatigue load increases.

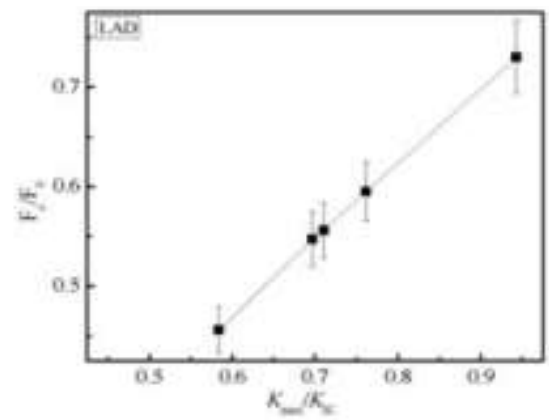

Fig. 2.Results in the anodic regime: LAD $(-500 \mathrm{mV}$ vs. SCE) 


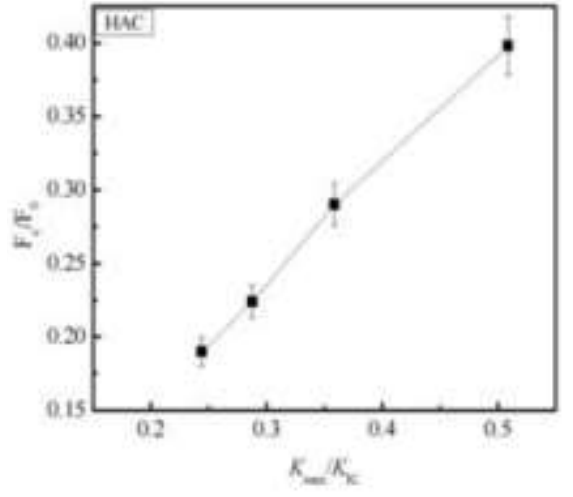

Fig. 3.Results in the anodic regime: HAC (-900mV vs. SCE)

These overload retardation effects are produced by the presence of compressive residual stresses in the vicinity of the crack tip as a consequence of the plastic zone created by the overload, surrounded by an elastic region which compresses the former. In this case the crack tip is in a certain sense pre-stressed by fatigue: the higher the overload level, the more pronounced the pre-stressing effect which delays the environmental damage process and improves the material performance in a corrosive environment. The appearance of compressive residual stresses in the near-tip region of a crack after cyclic loading has been analysed in general treatises on fatigue of materials (Suresh S. et al, 1991) and in technical papers (Rice JR. et al, 1967; Gortemaker P.C.M et al, 1981) dealing with the analyses of the stress-strain field in the vicinity of a crack tip after loading and unloading.

\section{Fractographic Examination}

A SEM fractographic analysis was carried out on the fracture surfaces of the samples, in order to detect any effect of residual stresses on the microscopical damage/fracture behaviour. Figure 4 shows SEM micrographs for the anodic regime (LAD, E $=-500 \mathrm{mV}$ SCE) and the maximum SIF level during the last stage of fatigue pre-cracking $\left(\mathrm{K}_{\max }=0.94 \mathrm{~K}_{\mathrm{IC}}\right.$. Fractographic analysis by the scanning electron microscopy (SEM) demonstrated the existence of transition topography between the fatigue pre-cracked and the cleavage like (unstable) fracture mode. For the anodic regime of LAD, such a topography is a sort of damage area ahead of the crack tip (Figure 4) and appears only in the most severe fatigue pre-cracking programmes $K_{\max }=0.94 K_{\text {IC }}$ ). Figure 5 offers SEM micrographs for the anodic regime (HAC, E=- $900 \mathrm{mV} \mathrm{SCE}$ ) and different values of the maximum SIF level during the last stage of fatigue precracking $\left(\mathrm{K}_{\max }=0.82 \mathrm{~K}_{\mathrm{IC}}\right)$. As in the case of HAC, microscopic topographies are shown in the vicinity of the crack tip, i.e., at the end of the fatigue pre-crack and the beginning of the environmentally assisted fracture area. Microscopic topographies are shown in the vicinity of the crack tip, i.e., at the end of the fatigue pre-crack and the beginning of the environmentally assisted fracture, in this hydrogen assisted area. Transition topography can be observed in almost all cases between the fatigue pre-crack and the cleavagelike unstable fracture mode with the exception of LAD in specimens pre-cracked under very low fatigue loads. Such a transition topography is caused by hydrogenassisted micro-tearing (TTS) in the case of HAC and mechanical damage - produced by the fatigue process in the case of LAD under high fatigue loads. For the cathodic regime of the HAC it is the so called tearing topography surface(TTS) in the vicinity of the crack tip(Figure 6) which appears in all fatigue pre-cracking programms, although its size is a decreasing the function of the $K_{\max }$ level, as shown in the Figure 6. TTS may be associated with hydrogen embrittlement (Thakur A. et al, 2007) processes in aluminum alloys.

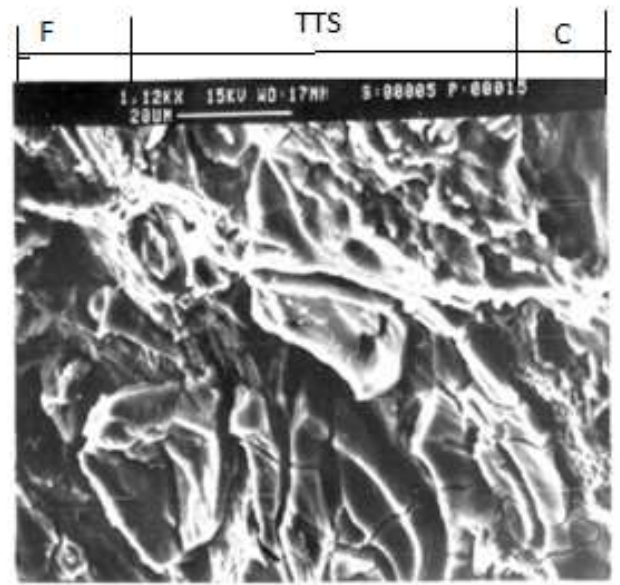

Fig. 4.Damaged area ahead of the crack tip (LAD, $\mathrm{K}_{\max }=$ $0.94 \mathrm{~K}_{\mathrm{IC}}$ )

F: Fatigue Pre-crack; TTS-Tearing Topographic Surface (Transition Topography); C: Cleavage-like (unstable fracture)

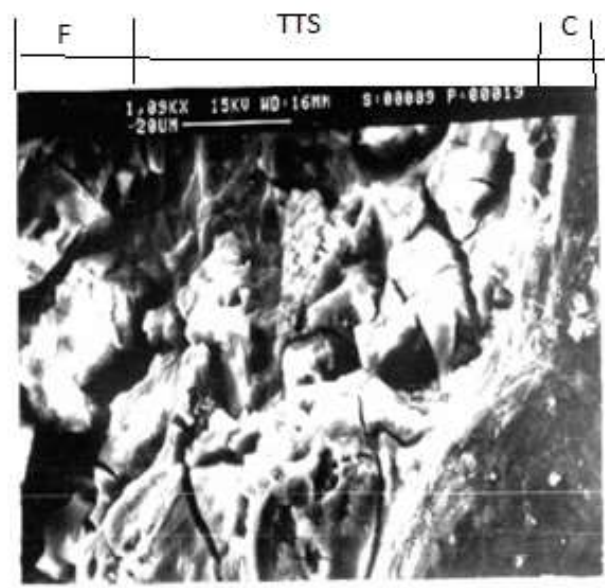

Fig. 5.Tearing topography surface ahead of the crack tip (HAC, $\mathrm{K}_{\max }=0.82 \mathrm{~K}_{\mathrm{IC}}$ )

F: Fatigue Pre-crack; TTS-Tearing Topographic Surface (Transition Topography); C: Cleavage-like (unstable fracture) 


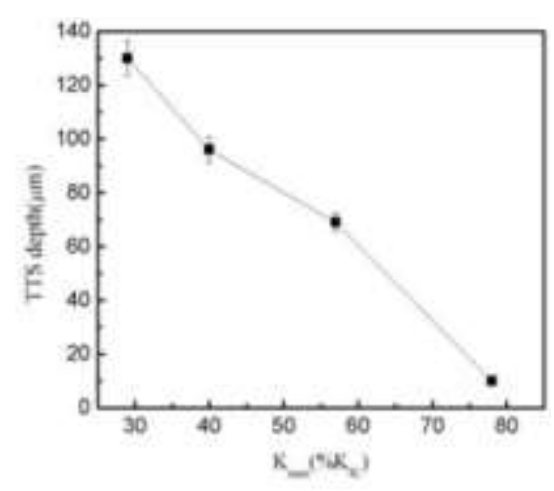

Fig. 6.TTS depth as a function of $K_{\max }$

These $K_{\max }$ effects are produced by the presence of the compressive residual stresses in the vicinity of the crack tip as a consequence of the cyclic plastic zone created by the fatigue pre-cracking procedure (cyclic residual stresses). The crack tip is in a certain sense pre-stressed by fatigue, the higher the overload level, the more pronounced the pre-stressing effect, which delays the environmental damage process (metal dissolution or hydrogen entry) and improves the material performance in a corrosive environment. The next section discusses several ways of modeling these cyclic residual stresses in the vicinity of the crack tip after loading and unloading the specimen.

\section{Modeling cyclic residual stress laws}

It would be useful to know the cyclic residual stress distribution ahead of the crack tip. There are three kinds of the difficulty in solving this problem. Firstly, from the theoretical point of view, it is impossible to know the exact stress distribution in the vicinity of the crack tip after fatigue pre-cracking (and therefore prior to the SCCtest), because the stress singularity is relaxed by the plastic zone spread. In addition, the numerical approach to the problem (finite element method, boundary integral equation method) is really complex due to the stress concentration and to the loading/unloading process.

Finally, the residual stress distribution after fatigue pre-cracking is only representative at the beginning of the SCC test, since it changes during it. From these considerations it seems that that the formulations of a simple model is sufficient indication of the residual stress distribution ahead of the crack tip after fatigue pre-cracking of the specimen.

A classical model of compressive residual stresses in the vicinity of the crack tip is that of Rice (Rice JR. et al, 1967), applicable to an elastic ideally plastic material under cyclic loading, and predictive of the residual stress distribution in the front of the crack tip at the end of final fatigue pre-cracking step, and therefore prior to a stress corrosion cracking or hydrogen embrittlement test. During this test, the residual stresses in front of the crack tip are redistributed in as the external load increases, and the compressions become tensions.
Distribution corresponding to $K=K_{\min }$ represents the residual stress state after acyclic loading/unloading process similar to that of fatigue pre-cracking (prior to any stress corrosion test with pre-cracked specimens) Moreover, the model is able to predict the depth of the maximum hydrostatic stress point, whose importance is determinant in HAC processes governed by stressassisted hydrogen diffusion.

The values of $\omega$ and $\triangle \omega$ represent the depth of the monotonic $\left(K_{\max }\right)$ and $\operatorname{cyclic}\left(K_{\min }\right)$ plastic zones, respectively, and their values are given by (RiceJR. et al, 1967):

$$
\begin{aligned}
& \omega=\frac{\pi}{2}\left\{\frac{K_{I C}}{\sigma_{y}}\right\}^{2} \\
& \Delta \omega=\frac{\pi}{32}\left\{\frac{\Delta K}{\sigma_{y}}\right\}^{2}
\end{aligned}
$$

Where $\sigma_{\mathrm{y}}$ is the yield strength of the material, $K_{\max }$ the maximum intensity factor during fatigue precracking(last step of loading, just prior to the SCC test) and $\Delta \mathrm{K}$ the stress intensity range in that $\operatorname{step}\left(\Delta K=K_{\max }{ }^{-}\right.$ $\left.K_{\text {min }}\right)$. In the tests performed in this work $K_{\min } \equiv 0$,and therefore $\Delta K \equiv K_{\max }$.

\section{Discussion}

In order to analyze the results of the SCC tests (either LAD or HAC) on the basis of the previous model, it is useful to estimate the plastic zone size during fatigue pre-cracking and at the end of the SCC test. Designating $\lambda_{f}$ as the dimensionless ratio of the maximum stress intensity factor during fatigue pre-cracking to the fracture toughness (which takes the values0.58, 0.69, $0.71,0.76$ and 0.94$)$ :

$\lambda_{f}=\frac{K_{\max }}{K_{I C}}$

We have:

$\omega_{f}=\frac{\pi}{8}\left\{\frac{K_{I C}}{\sigma_{y}}\right\}^{2} \lambda^{2} f$

$\triangle \omega=\frac{\pi}{32}\left\{\frac{K_{I C}}{\sigma_{y}}\right\}^{2} \lambda^{2} f$

In which $\omega_{\mathrm{f}}$ and $\Delta \omega_{\mathrm{f}}$ are respectively, the series of the monotonic $\left(\mathrm{K}=\mathrm{K}_{\max }\right)$ and cyclic $\left(\mathrm{K}=\mathrm{K}_{\min }\right)$ plastic zones during fatigue pre-cracking and the ratio $\left\{\frac{K_{I C}}{\sigma_{y}}\right\}$ is a characteristics of the materials.

In addition, a dimensionless ratio $\lambda_{c}$ of the critical stress intensity factor (at the end of the SCC test) to the fracture toughness in air may be defined as follows:

$\lambda_{c}=\frac{K_{C}(\text { SCCtest })}{K_{I C}(\text { air })}$ 
Which is approximately the ratio of the failure load in the solution to the failure load in air $\left(F_{C} / F_{O}\right)$ given in Figure 1 and Figure 2 (neglecting the sub-critical crack growth).Thusthe plastic zone size and the end of the SCC tests may be calculated as follows:

$\omega_{c}=\frac{\pi}{8}\left\{\frac{K_{I C}}{\sigma_{y}}\right\}^{2} \lambda^{2} c$

In accordance to the previous definitions, Figure 7 and Figure 8 show the plastic zone size during fatigue precracking (monotonic for $K=K_{\max }$ and cyclic for $K=K_{\min }=0$ ) and at the end of the SCC tests. For LAD phenomena(Figure 7) there is a strong protective effect caused by the fatigue pre-cracking load, and thus the final plastic zone during the LAD test surpasses clearly the cyclic and the monotonic plastic zone created during fatigue pre-cracking. The transition topography which appears in the microscopic fractographic analysis is probably created by the dissolution of the very strongly damaged zone close to the crack tip.

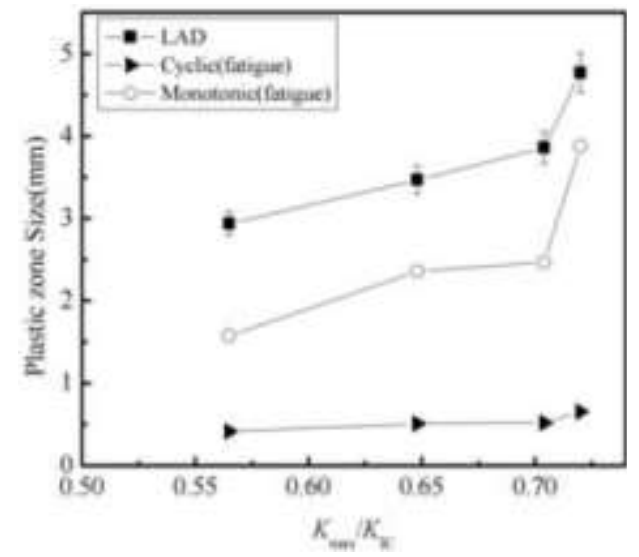

Fig. 7.Plastic zone size during fatigue pre-cracking (monotonic for $K=K_{\max }$ and cyclic for $K=K_{\min }$ ) and at the end of the LAD test

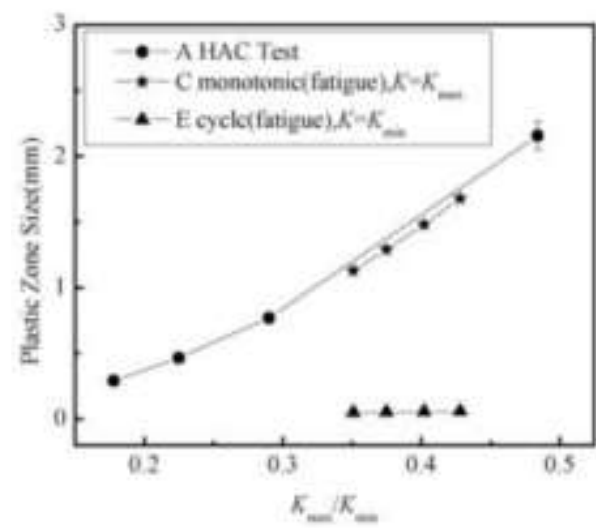

Fig. 8.Plastic zone size during fatigue pre-cracking (monotonic for $K=K_{\max }$ and cyclic for $K=K_{\min }$ ) at the end of the HAC test

As shown in the Figure 8, its size is related to the difference between the sizes of the plastic zone at the end of the HAC test and the monotonic plastic zone created by the fatigue maximum load, which seems to indicate that such a microscopic mode offracture- a sub-critical mode associated with hydrogen assisted micro-damage developsafter the previous tensile stress distribution is recovered. This explanation is consistent with a mechanism of the hydrogen transport by stress assisted hydrogen diffusion according to which hydrogen diffuses not only to the minimum concentration points, but also towards the maximum hydrostatic stress locations. Thus for $K_{\max }=0.94 K_{\text {IC }}$ the TTS size is negligible, the monotonic plastic zone created by fatigue is not exceeded during the HAC test and hydrogen penetration is impeded by this residual plastic zone.

\section{Conclusions}

1) Residual stresses generated in the vicinity of the crack tip during fatigue pre-cracking of specimens have shown to be the relevant in determining the material resistance to SCC.

2) The scanning electron microscopy of fracture surfaces reveals a major dependence of the cracking and/or pit severity on the applied stress intensity factor. $\mathrm{K}_{\mathrm{IC}}$ depends on the alloys.

3) Compressive residual stresses improve the SCC behavior and increase the failure loading an aggressive environment by delaying either the metal dissolution (in the case of LAD) or the hydrogen entry (in the case of HAC).

4) While HAC is extended over a process zone or fracture region (ITS) in the vicinity of the crack tip, LAD has a localized nature (metal dissolution at a single point). Though TTS values in both the case are not much difference for this alloy.

5) In HAC, Hydrogen transport by stress assisted hydrogen diffusion according to which hydrogen diffuses not only to the minimum concentration points, but also towards the maximum hydrostatic stress locations. $\mathrm{K}_{\max } / \mathrm{K}_{\min }$ and $\mathrm{K}_{\max } / \mathrm{K}_{\mathrm{IC}}$ offers a plastic zone sizes are little higher in LAD comparison to the HAC.

6) Microscopically, residual stresses produce a transition topography between the fatigue precrack and the cleavage-like (unstable) fracture mode. This transition zone is TTS-type for HAC $\left(0.82 \mathrm{~K}_{\mathrm{IC}}\right)$ and a kind of damaged region for LAD $\left(\mathrm{K}_{\mathrm{IC}}=0.94\right)$.

7) The Rice model of residual stress distribution in the vicinity of a crack tip may able toexplain the mechanical behavior of pre-cracked specimens in aggressive environments by estimating the stress corrosion tests.

\section{Acknowledgement}

I would like to express thank to Hindustan Aeronautics Limited for supplying research materials and IIT Bombay for allowing us to use their laboratory. 


\section{References}

BroekD. (1982), Elementary Engineering Fracture Mechanics, MartinusNijhoff, The Hague.

BarsomJ.M., and RolfeS.T.(1987), Fracture and Fatigue Control in Structures, Prentice-Hall, New Jersey.

SureshS. (1991), Fatigue of Materials, Cambridge University Press, 1991.

HanischA.H.andBurckL.H.(1982), Effects of overloads on the incubation time for stresscorrosion cracking of 7075 Aluminum. Corros.NACE, 38, 330-335.

ISO 7539-6 preparation and use of precracked specimens, 1989.

BranchoC.M., Radon J.C.andCulvervL.E.(1977), Influence of specimen orientation andloading history on SCC in aluminum alloy, Corrosion Science, 17, 125-141.

Bayoumim.R.(1996), The mechanics and mechanisms of fracture in stress corrosioncracking of aluminum alloys, Engineering Fracture Mechanics, 54(6),879-889.

Mulherinj.H.(1967), "Influence of environment on crack propagation characteristics ofhigh-strength aluminum alloys," Stress corrosion testing, ASTM STP 425, Am. Soc.Testing Mats., 66.
ToribioJ.andLancha A.M.(1996), Overload retardation effects on stress corrosion behavior ofprestressing steel. Construct Build Mater, 10,501-505.

RiceJR.(1967), Mechanics of crack tip deformation and extension by fatigue. ASTM STP 415,247-309.

GortemakerP.C.M, de PaterC., andSpieringR.M.E.J.(1981),Near crack tip finite strainanalysis. In Advances in Fracture Research (ICF5), ed. D. Francois, Pergamon,Oxford,151 160.

Thakur A. et al, Stress ratio and fatigue damage map study of hot rolled 7020-T6 aluminum alloy, Materials Science and EngineeringA,454-455, 2007, 552-557.

ParkinsR.N.(1993), Slow strain rate testing-25 years experience, ASTM STP 1210, 7-21.

ISO 7539-7(1989), Slow strain rate testing.

Thakur A. et al, Hydrogen embrittlement studies of aged and retrogressed-reaged $\mathrm{Al}-\mathrm{Zn}-\mathrm{Mg}$ alloys, Materials Chemistry and Physics,101, 2007, 441-447.

ToribioJ.(1993), The use of pre-cracked and notched slow strain rate specimens, ASTM STP 1210, 105-122. 